\title{
Biological therapy utilization, switching, and cost among patients with psoriasis: retrospective analysis of administrative databases in Southern Italy
}

This article was published in the following Dove Press journal:

ClinicoEconomics and Outcomes Research

\section{Francesca Guerriero Valentina Orlando Valeria Marina Monetti Veronica Russo Enrica Menditto \\ Center of Pharmacoeconomics (CIRFF), University of Naples Federico II, Naples, Italy}

Correspondence: Enrica Menditto CIRFF, Center of Pharmacoeconomics, University of Naples Federico II, Via Domenico Montesano 49, 80I3I Naples, Italy

Tel +39081678660

Fax +39081678658

Email enrica.menditto@unina.it

\begin{abstract}
Purpose: The aim was to describe the current use of biological therapies among patients affected by psoriasis and to analyze a drug utilization profile in naïve patients in terms of switching and treatment costs in a Local Health Unit (LHU) of Southern Italy.

Methods: We conducted an observational retrospective cohort analysis using the health-related administrative databases of a LHU in Southern Italy covering a population of about one million inhabitants. All subjects with a main or secondary diagnosis of psoriasis who received at least one prescription of biological therapies between January 1, 2010 and December 31, 2014 were analyzed. Switching rate was evaluated in naïve patients within the first year of treatment. Drug cost was calculated for all drugs prescribed and comprised both costs for psoriasis drugs and costs for other treatments.

Results: About $20 \%$ of patients identified with a diagnosis of psoriasis were under treatment with biological drugs. Among 385 subjects treated with biological therapy, 51.2\% were in treatment with etanercept and $33 \%$ with adalimumab. Among naïve patients, switching rate to a different biological drug, within the first year of treatment, was $7.3 \%$. The per patient yearly drug cost was $€ 10,536: 96.8 \%$ for psoriasis-related drugs and 3.2\% for other pharmaceutical treatments. The annual average cost per patient switching from the initial treatment was $€ 13,021$, while for those who did not switch from the initial treatment, the annual average cost was $€ 10,342$, with a significant difference of about $€ 2,680$ per patient per year $(p=0.002)$.

Conclusion: Our data may be useful in exploring the dynamics that characterize the use of biological therapy within a specific context and to optimize the use of resources for a better management of the disease.
\end{abstract}

Keywords: drug utilization, biological drugs, psoriasis, administrative databases, treatment cost, switch therapies

\section{Introduction}

Psoriasis is a chronic, noninfectious inflammatory skin disease, with a relapsing-remitting course, that impacts negatively on patients' quality of life. The most common form of the condition is psoriasis vulgaris or plaque psoriasis, which accounts for $80 \%$ of all cases. It is an underdiagnosed condition and consequently undertreated. About $2-4 \%$ of the population in western countries in Europe suffer from psoriasis, and its prevalence rate varies between $0.73 \%$ (in Scotland) and $2.9 \%$ (in Italy). ${ }^{1}$ Particularly in Italy, the prevalence is 3.1\% among adults and $2.15 \%$ among children. ${ }^{2}$ Psoriasis is associated with a higher risk of developing cardiovascular disease, 
psychiatric/psychologic issues, and psoriatic arthritis. The accurate etiology of psoriasis is still not completely understood, but a number of possible risk factors have been identified such as family history and environmental risk factors. In general, psoriasis is usually classified as mild, moderate, or severe, depending on the surface area affected, redness, and the thickness and esquamation of the plaques. Additionally, psoriasis imposes a substantial cost to health care systems as well as individuals. The economic burden of psoriasis is of particular concern given that this is a lifelong disease, and many patients require continuing treatment. Moreover, socioeconomic weight is attributable not only to direct but also to indirect costs, which represent a large proportion of total costs. . $^{3,4}$

Currently, psoriasis can be treated with topical agents, phototherapy, and systemic medications. ${ }^{1}$ The systemic treatments include conventional drugs such as ciclosporine and methotrexate and systemic biological therapies such as antitumor necrosis factor-alpha agents and anti-IL12/23 and anti-IL17 monoclonal antibodies. Biologic medications interfere in a selective way on various levels and with different actions on the pathological immunological processes that trigger and sustain psoriasis. In Italy, biologic drugs are reimbursed by National Health Service (NHS) in patients with an inadequate response to conventional systemic therapy or those who appear to have contraindications or who are intolerant to this kind of treatment. ${ }^{5,6}$

In the last decade, several new biological agents were licensed for clinical use in psoriatic patients substantially expanding the therapeutic armamentarium for its treatment. Although there is a wealth of information on the efficacy and safety of various treatments coming from randomized clinical trials, there is still need for more evidence from routine clinical practice on which patient is being prescribed which drug, and what impact this has on the health care system in terms of health care resource use and costs. Pattern of treatment and frequency of switch can help stakeholders to better understand differences among treatment options. This kind of information can support both physicians and payers in taking informed decisions on psoriasis management.

The aim of our study was to carry out, from a real-world prospective, a description of the current use of biological therapies among patients affected by psoriasis and to analyze a drug utilization profile in naïve patients in terms of switching and treatment costs in a Local Health Unit (LHU) of Southern Italy.

\section{Materials and methods \\ Study design}

We performed an observational retrospective cohort analysis according to the Strengthening the Reporting of Observational Studies in Epidemiology guidelines. ${ }^{7}$

\section{Data sources}

Fully anonymized data were extracted from the health-related administrative databases of a LHU in the Campania Region (Southern Italy) covering a population of about 1,000,000 inhabitants. These databases are complete and include validated data, and have been used in previous drug utilization studies. ${ }^{8-10}$

The data used for this study was obtained from: 1) civil registry containing demographic information (ie, age, gender, date of death, or emigration) of all residents covered by the Regional Health System; 2) pharmaceuticals database which contains, for all drugs dispensed by local pharmacies and reimbursed by the NHS, information regarding the patient identification code, drug code, dose, formulation, number of packages, date of prescription, date of dispensation, and drug price. Drugs are classified according to the Anatomical Therapeutic Chemical (ATC) classification system; ${ }^{11}$ 3) exemptions database, which contains the records of all disease exemptions, including the exemption code (identifying the disease for which the exemption was granted); and 4) hospital discharge database, which includes information on discharge for each hospitalization, the date of admission and discharge, main and up to five secondary diagnoses. Diagnoses are coded according to the International Classification of Diseases, Ninth Revision, Clinical Modification (ICD-9-CM).

All data sources were matched by record-linkage analysis through a unique and anonymous personal identification code. Because this automated system is anonymous, neither ethical committee approval nor informed consent was required. Furthermore, the anonymous data file is routinely used by the local health authority for epidemiological and administrative purposes. The reliability of this strategy to produce pharmacoepidemiological information has been previously documented. ${ }^{12,13}$

\section{Ethics statement}

According to the rules from Italian Medicines Agency (available at http://www.agenziafarmaco.gov.it/sites/default/ files/det_20marzo2008.pdf),23 retrospective studies using administrative data bases do not require ethics committee protocol approval; hence, the study was notified to the local ethics committee of Caserta LHU. 


\section{Definition of observation periods}

The study period spanned from January 1, 2010 to December 31, 2014 (Figure 1). The accrual period was defined as the period for ascertainment of exposure (incident users of biological drugs); it started 2 years after the start of the study period (ie, on January 1, 2012) and ended 1 year before the end of the study period (ie, on December 31, 2013). This allowed a 2-year look-back period preceding the first included biological drug prescription, to identify naïve patients (incident users of biological drugs), to describe prior medication use and comorbidities as well as a 1-year follow-up period after the last possible prescription of biological agents, to allow for adequate follow-up of patient outcomes (see Figure 1 for a graphical illustration of study period, accrual period and time frame for ascertainment of washout period, baseline characteristics, exposures, and outcomes).

\section{Study population and study drug categories}

The study population was selected among all health assisted patients who had registered on the databases during the study period and had at least 1 year of valid data. A patient selection flowchart is shown in Figure 2. All subjects with a primary or secondary diagnosis of psoriasis (ICD-9-CM code: 696.1) and/or disease exemption code (code: 045.69.6.1) between January 1, 2010 and December 31, 2014 were selected. All subjects with concomitant diagnosis of rheumatoid arthritis (ICD-9-CM code: 714), ankylosing spondylitis (ICD-9-CM code: 720.0), psoriatic arthritis (ICD-9-CM code: 696.0),
Crohn's disease (ICD-9-CM code: 555), and ulcerative colitis (ICD-9-CM code: 556) were excluded in order to avoid the potential bias of including in the analysis, as subjects might also have been taking one of the biological drugs under study for other chronic inflammatory diseases.

We included in the study all subjects with at least one prescription of biological therapies for the treatment of psoriasis. Specifically, the drugs under observation were: etanercept (ATC code: L04AB01), infliximab (ATC code: L04AB02), adalimumab (ATC code: L04AB04), and ustekinumab (ATC code: L04AC05). The date of first prescription of one of the drugs being observed in subjects with psoriasis, in the study period, was defined as the index date.

Naïve patients (incident users) were defined as subjects without prescriptions of biological drugs of interest during 24 months before the index date (washout period).

Subjects were observed from the index date for at least 12 months (follow-up period), and their clinical characteristics were investigated for 24 months prior to the index date (characterization period). Only patients with an observable follow-up of at least 12 months were considered in this study.

\section{Definitions and study outcome}

According to the ATC code used at index date, the drug utilization profiles, in terms of treatment costs and switching rate, of patients were estimated during the follow-up period.

A switch was defined as the presence of a different biological therapy (ATC code) other than that prescribed at the

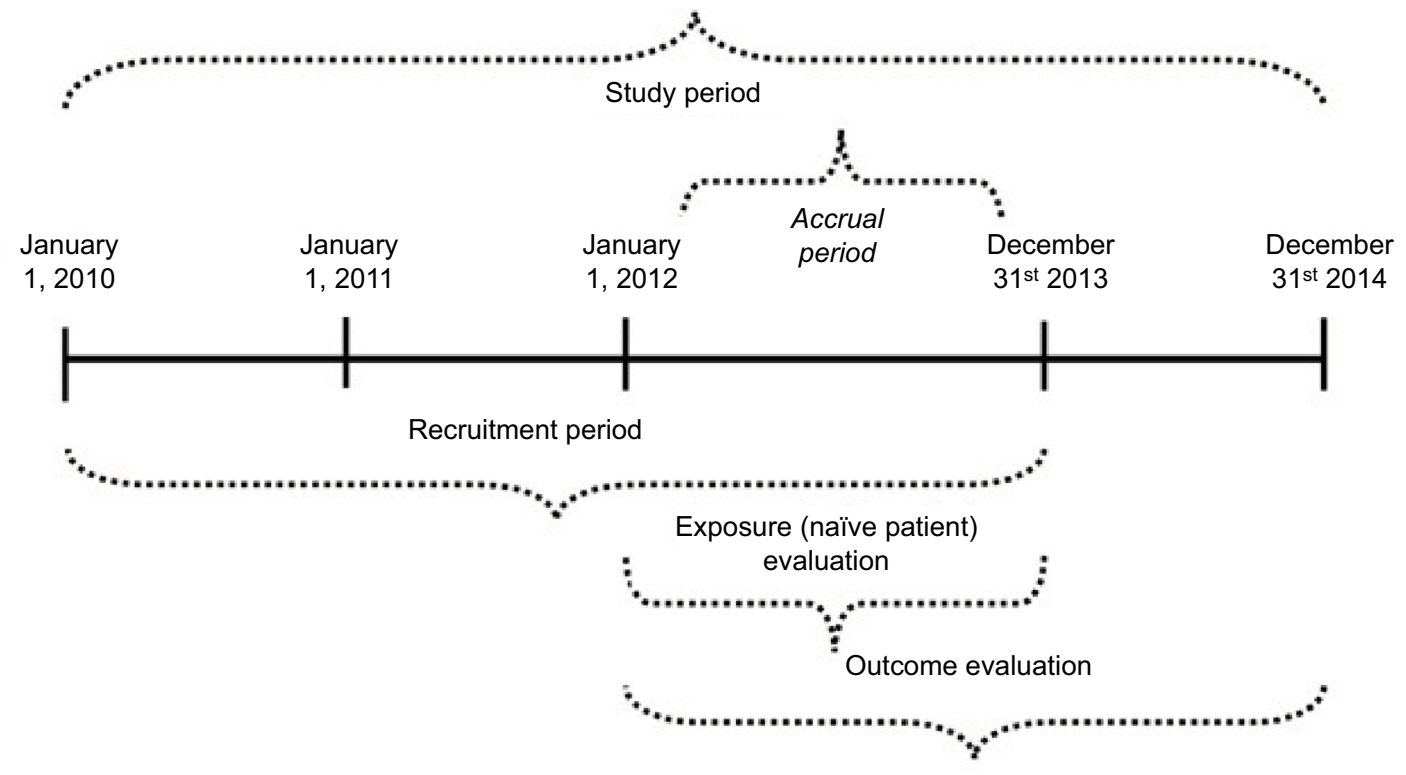

Figure I Definition of observation periods. 


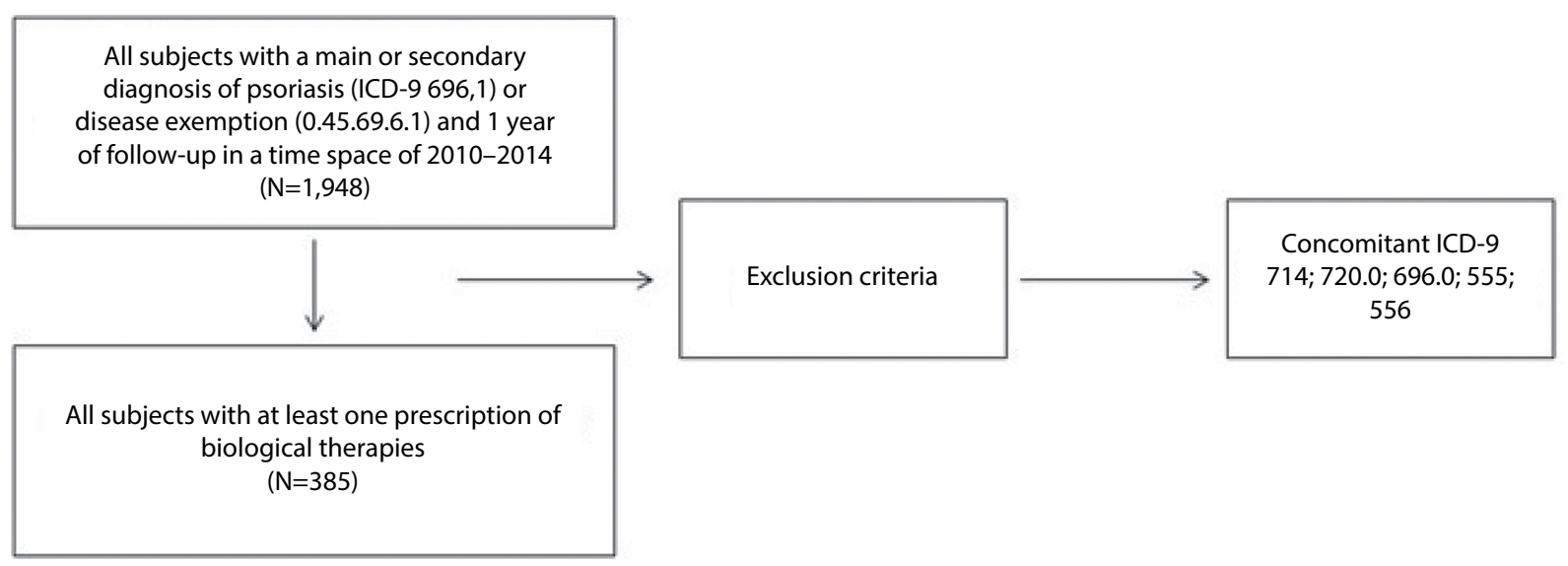

Figure 2 Sample selection flowchart.

Notes: ICD-9 7I 4 (Rheumatoid arthritis); ICD-9 720.0 (Ankylosing spondylitis); ICD-9 696.0 (Psoriatic arthritis); ICD-9 555 (Crohn's disease); ICD-9 556 (Ulcerative colitis). Abbreviation: ICD-9-CM, International Classification of Diseases, Ninth Revision, Clinical Modification.

index date during the follow-up period. Switching rate was calculated only for patients naïve to treatment.

Baseline information, including demographics characteristics, coprescribed drugs, and comorbidities, was collected. Polypharmacy was defined as a three-class variable: "excessive polypharmacy" was defined as the use of ten or more drugs; "polypharmacy" as the use of five to nine drugs; "non-polypharmacy" as the use of four or less drugs concomitantly. ${ }^{9}$ Comorbidity profiles were measured using the Charlson Comorbidity Index (CCI) that assigns a score to each concomitant disease identified through treatments and hospitalizations during the characterization period; $;^{10}$ the CCI score reflects patient's overall health status.

Subjects were characterized on the basis of coprescription of the following medications: diuretics drug (ATC code: C03); beta-blocking agents (ATC code: C07); calcium channel blockers (ATC code: C08); agents acting on the renninangiotensin system (ATC code: C09); lipid modifying agents (ATC code: C10); and antidiabetes drugs (ATC code: A10). Previous treatments with conventional systemic drugs (ATC codes: L01BA01, L04AD01, D05BB02) and topical agents (ATC codes: D05, D07) during 2 years before the data index date were evaluated.

\section{Cost of treatment}

The overall pharmaceutical consumption was assessed within 1 year from the index date and stratified according to ATC code at index date. Specifically, drug treatments related and unrelated to psoriasis were calculated. Drug consumption has been quantified in monetary terms in the prospective of the NHS. The total treatment cost was computed by multiplying the amount of drugs prescribed by unit cost. Drugs were priced according to the NHS's purchase price. Costs were expressed in euros $(€)$ currency and were assessed as an average cost per patient per year. The cost of each drug was calculated with reference to the time it was provided.

In the naïve patients, the mean and median annual pharmaceutical cost per patient was calculated separately as: 1) mean cost per patient with or without a switch from the initial treatment; 2) mean cost per patient switching from the initial treatment; and 3) mean cost per patient not switching from the initial treatment.

\section{Statistical analyses}

Characteristics of the study population were analyzed using descriptive statistics: quantitative variables were described by median, mean, and standard deviation $( \pm \mathrm{SD})$, while categorical variables were described by count and percentage. The significance of differences between mean costs was verified using nonparametric test for independent samples.

Linear regression analysis was also used to investigate the impact of sociodemographic and clinical characteristics on the dependent cost variables. All analyses were performed using SPSS software version 17.1 for Windows (SPSS Inc, Chicago, IL, USA).

\section{Results \\ Cohort characteristics and main outcome}

Overall, after applying selection criteria, 1,948 subjects with a diagnosis of psoriasis were identified. Among these, 385 
patients $(19.9 \%)$ were undergoing treatment with biologic drugs in the study period. By applying selection criteria, 126 of these subjects were identified as naïve patients.

The characteristics of cohort, stratified according to biological drug prescribed at index date, are reported in Table 1. The overall mean age (SD) of the subjects was $46.2( \pm 12.7)$ years and they were predominantly male $(58.4 \%)$.

During the study period, 197 (51.2\%) patients were under treatment with etanercept, 127 (33\%) patients with adalimumab, 42 (10.9\%) patients with ustekinumab, and $19(4.9 \%)$ patients with infliximab (Table 1). Of these patients on treatment with biological therapy, $48.8 \%$ received conventional systemic drugs and $38.7 \%$ received topical drugs.

The proportion of patients in polypharmacy, defined as the use of five to nine drugs, was $40.5 \%$ and was $23.6 \%$ in excessive polypharmacy, being defined as the use of more than ten drugs, as shown in Table 1. Switching rate within the first year of treatment was evaluated in naïve patients. About $8 \%$ of naïve patients switched to another biological drug. Average time to switch was 140 days. Switching rate stratified by ATC code at index date was as follows: $8.6 \%$ of patients starting with etanercept, $6.3 \%$ of patients starting with adalimumab, and $7.1 \%$ of patients starting with ustekinumab.

\section{Cost of treatment}

The average annual pharmaceutical costs stratified according to different biological drugs for the treatment of patients with psoriasis, based on drug consumption from index date and through the follow-up period, are reported in Table 2 . Figure 3 shows the mean of pharmaceutical cost stratified by ATC code. The per patient yearly drug costs on average were $€ 10,536$ of which $96.8 \%$ was cost of psoriasis-related drugs and $3.2 \%$ was related to other pharmaceutical treatments.

Table 2 shows the patient cost per year for patients switching from the initial treatment and for patients who do not switch from the initial treatment. The patient cost difference between the two groups was $€ 2,680$ ( $p=0.002)$. There was a

Table I Characteristics of patients in treatment with biologic drugs

\begin{tabular}{|c|c|c|c|c|c|}
\hline Characteristics & $\begin{array}{l}\text { Total } \\
N=385 \\
(100 \%)\end{array}$ & $\begin{array}{l}\text { Etanercept } \\
\mathrm{N}=197 \\
(51.2 \%)\end{array}$ & $\begin{array}{l}\text { Infliximab } \\
N=19 \\
(4.9 \%)\end{array}$ & $\begin{array}{l}\text { Adalimumb } \\
\mathrm{N}=127 \\
(33.0 \%)\end{array}$ & $\begin{array}{l}\text { Ustekinumab } \\
\mathrm{N}=42 \\
(\mathrm{I} 0.9 \%)\end{array}$ \\
\hline \multicolumn{6}{|l|}{ Gender } \\
\hline Female & $|6|(4 \mid .8)$ & $82(4 \mid .6)$ & $8(42.1)$ & $58(45.7)$ & $13(3 \mid .0)$ \\
\hline Male & $224(58.2)$ & II $15(58.4)$ & II (57.9) & $69(54.3)$ & $29(69.0)$ \\
\hline Age (mean \pm SD), years & $46.2(12.7)$ & $46.4(13.2)$ & $43.2(14.2)$ & $47.9(11.7)$ & $4 I .6(12.0)$ \\
\hline \multicolumn{6}{|l|}{ Charlson Index } \\
\hline 0 & $122(3 \mid .7)$ & $62(31.5)$ & $8(42.1)$ & $29(22.8)$ & $23(54.8)$ \\
\hline $1-4$ & $263(68.3)$ & $135(68.5)$ & II (57.9) & $98(77.2)$ & $19(45.2)$ \\
\hline Polypharmacy (mean \pm SD) & $6.9( \pm 4.8)$ & $6.7( \pm 4.6)$ & $9.7( \pm 7.0)$ & $7.1( \pm 4.6)$ & $6.2( \pm 4.6)$ \\
\hline $1-4$ & $138(35.8)$ & $70(35.5)$ & $7(36.8)$ & $45(35.4)$ & $16(38.1)$ \\
\hline $5-9$ & $156(40.5)$ & $83(42.1)$ & $3(15.8)$ & $51(40.2)$ & $19(45.2)$ \\
\hline$>10$ & $91(23.6)$ & $44(22.3)$ & $9(47.4)$ & $31(24.4)$ & $7(16.7)$ \\
\hline Diuretics & $4(1.0)$ & $2(1.0)$ & - & $\mathrm{I}(0.8)$ & $\mathrm{I}(2.4)$ \\
\hline Beta-blocking agents & $37(9.6)$ & $20(10.2)$ & $\mathrm{I}(5.3)$ & $13(10.2)$ & $3(7.1)$ \\
\hline Calcium channel blockers & $26(6.8)$ & $16(8.1)$ & - & $6(4.7)$ & $4(9.5)$ \\
\hline Agents acting on the renin-angiotensin system & $80(20.8)$ & $4 \mid(20.8)$ & $3(15.8)$ & $27(21.3)$ & $9(21.4)$ \\
\hline Lipid modifying agents & $54(14.0)$ & $27(13.7)$ & $\mathrm{I}(5.3)$ & $20(15.7)$ & $6(14.3)$ \\
\hline Drugs used in diabetes & $26(6.8)$ & $10(5.1)$ & $\mathrm{I}(5.3)$ & II (8.7) & $4(9.5)$ \\
\hline Treatment with systemic drugs & $149(38.7)$ & $76(38.6)$ & II (57.9) & $48(37.8)$ & $14(33.3)$ \\
\hline Treatment with topical drugs & $188(48.8)$ & $102(51.8)$ & $5(26.3)$ & $58(45.7)$ & $23(54.8)$ \\
\hline
\end{tabular}

Notes: Data presented as $\mathrm{N}(\%)$ unless otherwise indicated.

Table 2 Treatment cost per patient switching from the initial treatment and treatment cost per patient not switching from the initial treatment (cost per patient per year in euros)

\begin{tabular}{|c|c|c|c|c|c|}
\hline & \multicolumn{2}{|l|}{ Switch } & \multicolumn{2}{|l|}{ No switch } & \multirow[t]{3}{*}{$p$-value } \\
\hline & \multicolumn{2}{|c|}{ Cost per patient $(€)$} & \multicolumn{2}{|c|}{ Cost per patient $(€)$} & \\
\hline & Mean \pm SD & $\overline{\text { Median }}$ & Mean \pm SD & $\overline{\text { Median }}$ & \\
\hline Total cost & $13.02 \mid( \pm 4.317)$ & 13.237 & $10.342( \pm 3.898)$ & 10.535 & 0.002 \\
\hline Psoriasis-related drugs & $2.468( \pm 4.404)$ & $|2.81|$ & $10.024( \pm 3.939)$ & 10.268 & 0.006 \\
\hline Other drugs & $553( \pm \mathrm{I} .228)$ & 178 & $317( \pm 819)$ & 87 & 0.041 \\
\hline
\end{tabular}






Figure 3 Pharmaceutical cost stratified by ATC code.

Abbreviation: ATC, Anatomical Therapeutic Chemical

significant difference in median of total cost; psoriasis-related drugs cost; and other drug cost between switch and no switch sample ( $p=0.002 ; p=0.006 ; p=0.041$, respectively) for both psoriasis-related drugs and other drugs.

Linear regression analysis showed that switching therapy was a significant predictor of total pharmaceutical cost and psoriasis-related drug costs $(p<0.001)$ (Table 3$)$.

\section{Discussion}

This retrospective population-based drug utilization study evaluated the use of biological drugs in an Italian real-world setting among patients diagnosed with psoriasis and estimated pharmaceutical consumption and related cost using data from routine clinical practice.

Psoriasis therapy has progressed significantly over the last decade. Biological therapies are able to interfere in a highly selective manner, on various levels and with different action mechanism on the immunological processes which trigger and sustain psoriasis. According to what is planned for in Italy from the prescriptive and allowance conditions in the NHS system, at the time of analysis, biological drugs are indicated in patients with psoriasis as long as conditions of sufficient severity are present and it is impossible to carry out treatment using traditional methods. ${ }^{6}$

The principal findings of our analysis showed that about $20 \%$ of patients affected by psoriasis were treated with biological drugs. These results are in line with a recent survey conducted in five EU countries (including Italy) as well as US and Canada reporting that among patients with moderate-tosevere psoriasis, $\sim 20 \%$ of patients were receiving biological therapy. The survey, based on dermatologists' opinion, also
Table 3 Linear regression analysis of the treatment costs

\begin{tabular}{|c|c|c|c|c|c|}
\hline \multirow[t]{2}{*}{ Variables } & \multirow[t]{2}{*}{ St. $\beta$} & \multirow[t]{2}{*}{$p$-value } & \multirow[t]{2}{*}{$\beta$-value } & \multicolumn{2}{|l|}{$95 \% \mathrm{Cl}$} \\
\hline & & & & Lower & Upper \\
\hline \multicolumn{6}{|l|}{ Total cost } \\
\hline Switch & 0.179 & $0.00 I^{*}$ & $2.744,736$ & I.188, 303 & $4.301,170$ \\
\hline Gender & -0.007 & 0.887 & $-58,297$ & $-86 I, 497$ & 744,903 \\
\hline Age & 0.064 & 0.653 & 20,110 & $-67,749$ & 107,970 \\
\hline Polypharmacy & -0.024 & 0.659 & $-126,688$ & $-691,068$ & 437,692 \\
\hline Charlson Index & -0.044 & 0.757 & 166,296 & $-888,311$ & $1.220,903$ \\
\hline \multicolumn{6}{|l|}{$\begin{array}{l}\text { Psoriasis- } \\
\text { related drug } \\
\text { costs }\end{array}$} \\
\hline Switch & 0.174 & $0.001 *$ & $2.684,729$ & $1.109,195$ & $4.260,263$ \\
\hline Gender & 0.009 & 0.860 & 72,777 & $-740,279$ & 885,834 \\
\hline Age & 0.065 & 0.650 & 20,526 & $-68,412$ & 109,463 \\
\hline Polypharmacy & -0.079 & 0.152 & $-417,069$ & $-988,375$ & 154,236 \\
\hline Charlson Index & 0.021 & 0.886 & 78,069 & $-989,480$ & $1.145,619$ \\
\hline \multicolumn{6}{|l|}{ Other drugs } \\
\hline Switch & 0.018 & 0.716 & 60,012 & $-263,829$ & 383,853 \\
\hline Gender & -0.076 & 0.124 & $-131,076$ & $-298,194$ & 36,043 \\
\hline Age & -0.006 & 0.964 & $-0,416$ & $-18,696$ & 17,865 \\
\hline Polypharmacy & 0.259 & $0.000 *$ & 290,386 & 172,957 & 407,814 \\
\hline Charlson Index & 0.110 & 0.430 & 88,227 & $-|3|, 20 \mid$ & 307,655 \\
\hline
\end{tabular}

Note: ${ }^{*} p$-value $<0.05$ was considered to be statistically significant.

recognized that psoriasis is undertreated and that there is an unmet treatment need for patients with psoriasis. ${ }^{14}$ Our analysis showed that, among patients in treatment with biologics, $51.4 \%$ are treated with etanercept, $32.8 \%$ with adalimumab, and $10.9 \%$ with ustekinumab. Other Italian studies based on data coming from specialized centers participating in the Psocare network reported percentages of use for etanercept and adalimumab in line with our figures..$^{15}$ Both US retrospective studies, using large claims database, ${ }^{16}$ and a UK retrospective study ${ }^{17}$ reported similar rates for ustekinumab use, but contrary, higher use of adalimumab.

After 1 year of observation, we detected a switching rate of about $8 \%$. The results presented in our study are consistent with other analyses conducted in the real-world settings. ${ }^{18}$ The median length of time that switched patients continued their index therapy was 140 days.

Our analysis also assessed the pharmaceutical cost for psoriasis in patients treated with biologics. The mean cost of treatment per patient per year was $€ 10,536$. This result is in line with other analysis performed in real practice settings in Italy. ${ }^{19}$ Patients switching as early as the first year of follow-up had higher treatment costs than patients who do not switch ( $€ 13,021 \mathrm{vs} € 10,342, p=0.002)$. This finding suggests that clinical conditions were likely deteriorating (higher consumption of medicinal products) and/or patients had switched to a different pharmacological therapy that was more expensive than the previous one. 
Certainly, the financial impact in biological therapies is notable. In the NHS, biologic drugs amount to $€ 30.1$ per capita $(13.7 \%$ of the Italian NHS pharmaceutical expenditure). In particular, the products considered in the analysis represent $28.9 \%$ of the expenditure for biologic drugs. ${ }^{15}$ Indeed, they have transformed the treatment of psoriasis.

The recent introduction of biosimilar drugs in the therapeutic armamentarium for the treatment of psoriasis could reduce costs while increasing access to biologic medications. ${ }^{14,20-22}$ On the other hand, the arrival of new, more selective and promising molecules in terms of efficacy has also produced changes in outcome, which are more ambitious than in the past, which could have positive effects on improvement in the efficiency of treatment with a reduction in expenses in other sectors.

Italian opinion leaders have the feeling that there is an excessively conservative approach to treatment by local operators. The tendency appears to be, in fact, that of treating moderate psoriasis as light and that of a serious nature as being moderate, preferring topical drugs and using lengthy conventional treatments. ${ }^{22}$ Decision makers should be aware that a patient treated with traditional drugs who is unresponsive contributes to a higher cost to the community (working capacity, necessary assistance, added treatment, morbidity) than a responsive patient being treated with biological products.

We feel that the main strength of our analysis lies in providing an overview of the use and consumption of biologics in a real setting. This is useful in exploring the dynamics that are currently characterizing the use of biological therapy within a specific context. To our knowledge this is the first study exploring switching rates and related costs in patients with psoriasis treated with biological drugs in the Italian setting.

Our estimates of the prescription patterns for biologics among subjects with psoriasis exhibit some similarities and differences from what has been reported in literature. However, it is difficult to directly compare estimates because differences in study populations may explain some observed variations.

As a retrospective administrative database study, details on treatment response, side effects, and reasons for switching or gaps in treatment were unavailable. As such, we were unable to determine if treatment switching was as a result of adverse effect or loss of response. Although rigorous, our measurements are also subject to some limitations such as, for instance, we did not have information of psoriasis severity beyond the fact that biologics are indicated for moderateto-severe disease. In order to prevent bias in the results of our analysis, we excluded patients with other chronic inflammatory diseases, who might also have been taking one of the biological drugs under study but for a different indication of use than for psoriasis.

The results in our real-life study, even if referred to a limited sample, are indicative of the opportunity for improvement in the use of resources by the NHS because the knowledge of the prescriptive scheme and the use of resources can stimulate the planning of health actions with the aim of strengthening the proposed services for its treatment.

The cost analysis considered drug costs at the moment of the analysis. These costs could be dropped with the introduction of biosimilars on the market.

Further studies are needed to explore factors influencing switching from and the discontinuation of therapy and possible transferal features of expenditure.

\section{Conclusion}

Psoriasis is a complex chronic disease, and appropriate management is correspondingly complex.

Our study provided a snapshot of drug utilization patterns of biologics in psoriasis treatment. Patients switching as early as the first year of follow-up had higher treatment costs than patients who did not switch. Providing information based on real-world data may be useful to explore the dynamics that characterize the use of biological therapy within a specific context and to optimize the use of resources for a better management of the disease.

\section{Acknowledgment}

We thank Dr.ssa Francesca Colaccio from ASL Caserta for her support in data acquisition and manuscript revision.

\section{Author contributions}

All authors contributed toward data analysis, drafting and critically revising the paper and agree to be accountable for all aspects of the work.

\section{Disclosure}

The authors report no conflicts of interest in this work.

\section{References}

1. Parisi R, Symmons DPM, Griffiths CEM, et al; on behalf of the Identification and Management of Psoriasis and Associated Comorbidity (IMPACT) project team. Global epidemiology of psoriasis: a systematic review of incidence and prevalence. $J$ Invest Dermatol. 2013; 133:377-385.

2. Saraceno R, Mannheimer R, Chimenti S. Regional distribution of psoriasis in Italy. J Eur Acad Dermatol Venereol. 2008;22:324-329.

3. World Health Organization. Global report on psoriasis. World Health Organization; 2016. Available from: http://apps.who.int/iris/bitstr eam/10665/204417/1/9789241565189_eng.pdf. Accessed May 15, 2017. 
4. Brezinski EA, Dhillon JS, Armstrong AW. Economic burden of psoriasis in the United States: a systematic review. JAMA Dermatol. 2015;151(6):651-658.

5. Menter A, Griffiths CEM. Current and future management of psoriasis. The Lancet. 2007;370(9583):272-284.

6. Humira (adalimumab) prescribing information. European Medicines Agency. Available from: http://www.ema.europa.eu/ema/index. jsp?curl=pages/medicines/human/medicines/0081/human_med_000822. jsp\&mid=WC0b01ac058001d124. Accessed June 28, 2014.

7. STROBE Statement - Checklist of items that should be included in reports of cohort studies. Available from: www.strobe-statement.org/. Accessed May 15, 2017.

8. Iolascon G, Gimigliano F, Orlando V, Capaldo A, Di Somma C, Menditto E. Osteoporosis drugs in real-world clinical practice: an analysis of persistence. Aging Clin Exp Res. 2013;25(1):S137-S141.

9. Casula M, Catapano AL, Piccinelli R, et al. Assessment and potential determinants of compliance and persistence to antiosteoporosis therapy in Italy. Am J Manag Care. 2014;20(5):e138-145.

10. Orlando V, Guerriero F, Putignano D, et al. Prescription patterns of antidiabetic treatment in the elderly. Results from Southern Italy. Curr Diabetes Rev. 2015;12(2):100-106.

11. World Health Organization Collaborating Centre for Drug Statistics Methodology. Available from: http://www.whocc.no/atc_ddd_index/. Accessed December 19, 2014

12. Cammarota S, Bruzzese D, Sarnelli G, et al. Proton pump inhibitors prescribing following the introduction of generic drugs. Eur J Clin Invest. 2012;42(10):1068-1078.

13. Cammarota S, Bruzzese D, Catapano AL, et al. Lower incidence of macrovascular complications in patients on insulin glargine versus those on basal human insulins: a population-based cohort study in Italy. Nutr Metab Cardiovasc Dis. 2014;24(1):10-17.

14. Van de Kerkhof PC, Reich K, Kavanaugh A, et al. Physician perspectives in the management of psoriasis and psoriatic arthritis: results from the population-based Multinational Assessment of Psoriasis and Psoriatic Arthritis survey. J Eur Acad Dermatol Venereol. 2015;29:2002-2010.

15. Spandonaro F, Ayala F, Berardesca E, et al. The cost effectiveness of biologic therapy for the treatment of chronic plaque psoriasis in real practice settings in Italy. Bio Drugs. 2014;28(3):285-295.
16. Feldman SR, Zhao Y, Zhou H, Herrera V, Tian H, LiY. Economic impact of above-label dosing with etanercept, adalimumab, or ustekinumab in patients with psoriasis. J Manag Care Spec Pharm. 2017;23(5): 583-589.

17. Iskandar IYK, Ashcroft DM, Warren RB, et al. Demographics and disease characteristics of patients with psoriasis enrolled in the British Association of Dermatologists Biologic Interventions Register. $\mathrm{Br} J$ Dermatol. 2015;173(2):510-518.

18. Doshi JA, Takeshita J, Pinto L, et al. Biologic therapy adherence, discontinuation, switching, and restarting among patients with psoriasis in the US Medicare population. J Am Acad Dermatol. 2016;74(6): 1057-1065.

19. DETERMINA 9 dicembre 2014 - Regime di rimborsabilità e prezzo del medicinale per uso umano «Remsima» (infliximab), autorizzata con procedura centralizzata europea dalla Commissione Europea. (Determina n. 1471/2014). (14A09840) (GU Serie Generale n.302 del 31-12-2014). [DETERMINATION 9 December 2014 - Redemption and price regime of the medicinal product for human use "Remsima" (infliximab), authorized with a centralized European procedure by the European Commission. (Determine No. 1471/2014). (14A09840) (GU Series General No.302 of 31-12-2014)]. Available from: http://www. gazzettaufficiale.it/eli/gu/2014/12/31/302/sg/pdf. Accessed May 15, 2017. Italian.

20. Polistena B, Calzavara-Pinton P, Altomare G, et al. The impact of biologic therapy in chronic plaque psoriasis from a societal perspective: an analysis based on Italian actual clinical practice. J Eur Acad Dermatol Venereol. 2015;29(12):2411-2416.

21. Feldman SR, Burudpakdee C, Gala S, et al. The economic burden of psoriasis: a systematic literature review. Expert Rev Pharmacoecon Outcomes Res. 2014;14:685-705.

22. Feldman SR, Goffe B, Rice G, et al. The challenge of managing psoriasis: unmet medical needs and stakeholder perspectives. Am Health Drug Benefits. 2016;9(9):504.

23. Gazzetta Ufficiale della Repubblica Italiana, Determinazione del 20 marzo 2008. [Official Gazette of the Italian Republic, Determination of the 20th March 2008. Available at http://ww.gazzettaufficiale.it/atto/ stampa/serie_generale/originario. Accessed May 15, 2017. Italian.
ClinicoEconomics and Outcomes Research

\section{Publish your work in this journal}

ClinicoEconomics and Outcomes Research is an international, peerreviewed open-access journal focusing on health technology assessment, pharmacoeconomics and outcomes research in the areas of diagnosis, medical devices, and clinical, surgical and pharmacological intervention. The economic impact of health policy and health systems

\section{Dovepress}

organization also constitute important areas of coverage. The manuscript management system is completely online and includes a very quick and fair peer-review system, which is all easy to use. Visit http://www.dovepress.com/testimonials.php to read real quotes from published authors. 